\title{
Effects of Biaxial Ankle Strengthening on Muscle Efficiency during Gait in Chronic Stroke Patients: A Randomized Controlled Pilot Study
}

\author{
Ji-Eun Cho \\ National Rehabilitation Center \\ Wan-Hee Lee \\ Samyook University \\ Joon-Ho Shin \\ National Rehabilitation Center
}

Hogene Kim ( $\square$ hogenekim@korea.kr)

\section{Research}

Keywords: Ankle, Strengthening, Coactivation, Feedback, Gait, Stroke

Posted Date: April 2nd, 2020

DOI: https://doi.org/10.21203/rs.3.rs-19660/v1

License: (c) (1) This work is licensed under a Creative Commons Attribution 4.0 International License. Read Full License 


\section{Abstract}

Background: Ankle dysfunction in stroke patients is a common but serious cause of balance and gait impairment. However there seldom exists comprehensive paretic ankle training. Thus we investigated the effects of biaxial ankle muscle training (AMT) using visual feedback as a means to improve ankle strength and functional activities in stroke patients.

Methods: The study design was a randomized controlled pilot trial with concealed allocation and assessor blinding, and intention-to-treat analysis. Twenty-five patients with stroke under inpatient rehabilitation with difficulty walking (e.g. foot drop), or ankle muscle weakness participated. The experimental group underwent AMT consisting of passive stretching, contraction to match movement, and active-resistive strengthening using visual feedback for 40 minutes per day, 5 times per week for 4 weeks. The control group underwent ankle-related physical therapy including ankle range of motion(ROM) exercises for matched time period. The outcomes were ankle isometric contraction force, Fugl-Meyer lower extremity (FM-L), Berg balance scale (BBS), walking speed, and ankle co-contraction index $(\mathrm{Cl})$ during gai.

Results: The results showed significant between-group differences for ankle isometric contraction in each direction $(P<0.05), F M-L(P<0.01)$ and stance-phase $\mathrm{Cl}(P<0.05)$. Post training, the AMT group displayed significant differences in the ankle isometric contraction in each direction $(P<0.01)$, ankle proprioception $(P<0.05)$, and walking speed $(P<0.05)$.

Conclusions: Our findings demonstrate the significant short-term effects of AMT on ankle strength, walking speed, and ankle muscle efficiency in chronic stroke survivors.

\section{Background}

Ankle dysfunction in stroke patients is a common but serious cause of balance and gait impairment [1]. Ankle impairments in patients with chronic stroke, including weakness of dorsiflexor, spasticity of plantarflexor, passive stiffness of plantarflexors, and ankle joint pathology, are more prominent than impairments of other proximal lower extremities and inhibit daily activities long-term [2]. These impairments in stroke patients require continuous ankle-foot orthosis after discharge, which result in reduced walking speed, asymmetric gait pattern, and increased risk of falls $[3,4]$.

Ankle muscle weakness and sensory impairments cause difficulties in ankle motor control during a gait sequence. First, weakness in all muscles surrounding the ankle joint is a common symptom. For instance, weakness of the ankle plantarflexor reduces the mechanical push-off [5] and weakness in the ankle dorsiflexor and evertor causes foot drop and abnormal muscle co-contraction during the swing phase of the paretic side [6]. In addition, contraction of the ankle muscles requires coordination of the agonist, antagonist and synergist muscles. Second, sensory functions such as touch, joint sensation, and proprioception are important factors for the quality of movement. Approximately one-third of stroke patients reported impairment in proprioception, which makes it difficult to retrain normal movements [7], 
even when repeatedly performing functional movements [8]. Therefore, an ankle intervention that integrates motor (strength) and sensory (proprioception) functions for retraining the functional movements of stroke patients may be helpful.

Recently, rehabilitative intervention using robotic technology has been performed to improve the hemiparetic gait in stroke patients. Generally, robot-assisted gait training is not effective for gait quality but for gait speed and aerobic function improvement through repetitive gait pattern re-education $[9,10]$. However, chronic stroke patients with activities that require community walking and difficult task-solving skills, including walking on rugged surfaces and ramps, require a fundamental solution for their deficient ankle function. A recent study reported that intensive task-specific training of impaired joints is effective in learning neuromuscular movements [11]. An example is Anklebot, which performs repetitive biaxial ankle movements based on a game in sitting position. These ankle movement training methods showed improvements in ankle motor control, gait speed, and gait quality, including paretic step length and gait symmetry in stroke patients [12-14]. However, one Anklebot study showed effectiveness only for individuals with moderate to high functional level, i.e., individuals who can at least move their ankles by themselves [15]. Because the impaired central nervous system in stroke survivors can affect both peripheral sensory and motor functions, comprehensive ankle rehabilitative intervention is required. Use of various measurements (such as electromyography) to quantitatively assess which ankle actually contributes during walking will help to understand the effects of ankle rehabilitation during walking.

For the targeted and comprehensive ankle training for general stroke hemiparesis, we developed an ankle muscle training (AMT) method using a custom-made ankle device that applies 3 steps: passive stretching, muscle contraction to match movement, and active-resistive strengthening. The design of ankle device reflects the anatomical characteristics of the ankle and a range of movements, from diagonal movement to simple biaxial movement, are included to reproduce actual ankle motion. A previous study using this device reported that passive bi-axial ankle stretching training decreased hemiparetic stiffness and improved walking performances on uneven terrain [16].

Therefore, the aim of this study was to study the effects of AMT on ankle strength, balance ability, walking speed, and ankle muscle efficiency during a gait cycle in patients with chronic stroke. We hypothesized that the proposed AMT will affect ankle strength in chronic stroke patients and that altered ankle strength would improve functional activities including balance and gait.

\section{Methods}

\section{Design}

A single blinded, parallel-group, randomized controlled trial with blinding of assessors and concealed allocation was conducted. A person not involved in the trial created a blocked random allocation scheduled for 20 participants using Microsoft Excel. Blocking ensured equal numbers of participants in the experimental and control groups. Participants in the experimental group underwent ankle movement 
training (AMT) consisting of passive stretching, contraction to match movement, and active-resistive strengthening using visual feedback for four weeks. Participants in control group underwent usual ankle relative physical therapy for four weeks. All participants were re-assessed at the end of the four-week intervention period (Fig. 1). All applicable institutional and governmental regulations concerning the ethical use of human volunteers were followed.

\section{Participants}

Participants were recruited from the National Rehabilitation Center's hospital between July and October 2018. The following eligibility criteria were applied: (1) chronic post-stroke hemiparesis; (2) weakness of ankle muscles, defined according to the medical research council (MRC) scale (strength range grade 1 to 4); (3) independent walking with ankle impairment such as foot drop (functional ambulatory category score > 3 [17]). The following exclusion criteria were applied: (1) complications of orthopedic disorders; (2) cognitive impairment (mini-mental state examination score $\leq 24)$; (3) insufficient visual acuity to see a screen (e.g. diplopia). This study was approved by the Institutional Review Board of the National Rehabilitation Center and participants provided informed consent before data collection began.

\section{Intervention}

In this study, AMT was performed using a published ankle training device and settings [16]. A monitor providing visual feedback was placed at eye level of the participant (Fig. 2-B). The AMT protocol consisted of three steps (Fig. 2-A). In the first step (passive stretching training) the maximum passive ankle range of motion (ROM) was measured, and passive stretching training through repetitive ROM exercise according to the movement direction was performed. In the second step (muscle contraction training to match movement), the current and target positions of the ankle were provided through a screen. When the ankle was passively moved from the original position to the target position by the robotic device, participants actively contracted their ankle muscles to enable movement towards the target position. A green light indicated the coincidence between robotic passive movement and participant's active movement. In the final step (active-resistive strengthening training), participants actively moved their ankle from the original position to the target position without an assistance force. To improve fine movements and muscle strengthening, different target sizes and progressive resistance thresholds were applied. Participants had been allocated up to 30 seconds per target and were allowed to fully rest for 5 seconds to alleviate muscle fatigue. All training was performed within $80 \%$ of the individual maximum ankle ROM. AMT was performed for 40 minutes per day, 5 times per week for 4 weeks.

Participants in control group performed general physical therapy related to the ankle. Ankle ROM exercise with assistance and functional electrical stimulation provided to the affected quadriceps and tibialis anterior muscles on alternating occasions, including resting periods, and gait training with assistance were performed for 40 minutes per day, 5 days per week for 4 weeks.

\section{Outcome measurements}


All outcome measurements were collected by trained therapists, who were blinded to group allocation. Baseline measurements were collected prior to randomization (Week 0). After four weeks, interventions were ceased and outcome measurements for both groups were collected (Week 4). Further details about the outcome measurements are provided below.

The isometric contraction force of the paretic ankle was measured using a portable manual muscle strength tester (Lafayette, USA, 2018). The isometric strength of the ankle dorsiflexor, plantarflexor, invertor, and evertor was measured for 5 seconds, and the maximum value was recorded. Using a position matching test [18], ankle proprioception was evaluated as the difference between the actual movement angle and the remembered angle. Ankle proprioception was measured using the device moving at constant velocities $\left(2.14^{\circ} / \mathrm{s}\right)$ with passive movement [18]. To avoid other environmental stimuli, participants wore eye masks and earplugs.

To measure motor impairment, the motor domain of FM-L was used. The motor domain included measurements of movement, coordination, and reflex action for the hip, knee, and ankle [19].

As balance-related measurements, BBS enforces individuals to maintain a static position, shift their center of mass with respect to their support and diminish the base of support [20].

For walking speed evaluations, participants repeated 10-meter walking test (10MWT) trials at least four times on a $1.5 \bigotimes 15 \mathrm{~m}$ walkway covered with industrial carpeting at a comfortable speed.

The ankle muscle co-contraction index $(\mathrm{Cl})$ was measured for ankle muscle efficiency during a gait sequence using a wireless electromyogram (Delsys Trigno Wireless EMG, Delsys, USA, 1993). The sensor was attached to both sides of the tibialis anterior and medial gastrocnemius. The sampling frequency was set to $2000 \mathrm{~Hz}$, and a Butterworth bandpass filter of $10-350 \mathrm{~Hz}$ and full-wave rectification were applied. We then calculated the root mean square of the signal with a $100 \mathrm{~ms}$ window for analysis. To normalize the collected muscle activity data according to the walking cycle, \%RVC for maximum EMG in the walking cycle was calculated. Calculation of $\mathrm{Cl}$ was based on a previous study that compared the activity of the antagonist and agonist muscles divided into the stance phase and swing phase as follows [21].

$$
C I=\frac{\int_{t 1}^{t_{2}} E M G_{T A}(t) d t}{\int_{t_{1}}^{t_{2}}\left[E M G_{T A}+E M G_{M G}\right](t) d t} \times 100
$$

\section{Data Analysis}

Sample size was calculated according to a previous study that reported the effect of ankle training following chronic stroke [22]. Calculation was based on the pretest-posttest gait velocity for the experimental group, using an alpha of 0.05 at $95 \%$ power. The total sample size was determined to be 10 
for the separate groups (effect size: 1.062 , actual power: 0.964 ). The program used was $G$ * Power 3.1.9.2.

All statistical analyses were performed using SPSS ver. 21.0 (SPSS for Windows; SPSS Inc., Chicago, IL, USA). The results are presented as the mean \pm standard deviation. Prior to all analyses, the normality of data was assessed with a one-sample Kolmogorov-Smirnov test. Comparisons of the changes between the two groups were performed using independent $t$-tests and within groups using a paired t-test. For all tests, statistical significance was set at 0.05 .

\section{Results}

\section{Participants}

Thirty participants underwent pre-enrollment screening evaluation. A total of 25 participants, 13 in the experimental group and 12 in the control group, who met the inclusion criteria were included in this study. At baseline, no significant differences were found between groups for demographic data or measurements (Table 1). Two individuals in the control group dropped out due to discharge during the training. Therefore, post-testing and analysis were completed for 13 individuals in the AMT group and 10 in the control group. 
Table 1

Participant characteristics at baseline

\begin{tabular}{|llll|}
\hline & AMT group (n=13) & $\begin{array}{l}\text { Control group } \\
(\mathbf{n}=10)\end{array}$ & P value \\
\hline Age (y) & $58.0(6.5)$ & $57.2(9.7)$ & 0.837 \\
\hline Sex (M/F) & $10 / 3$ & $8 / 2$ & 0.912 \\
\hline Weight (kg) & $68.9(7.8)$ & $68.1(9.39)$ & 0.836 \\
\hline Height (m) & $171.4(6.9)$ & $168.6(8.7)$ & 0.443 \\
\hline Time post-stroke (months) & $10.9(6.5)$ & $10.6(3.3)$ & 0.905 \\
\hline Stroke side (R/L) & $5 / 8$ & $4 / 6$ & 0.821 \\
\hline Modified Ashworth Scale (0/1/1+) & $1 / 3 / 6$ & $1 / 3 / 9$ & 0.723 \\
\hline Functional Ambulation Category (0-5) & $4.6(1.0)$ & $4.5(0.5)$ & 0.885 \\
\hline K-MMES (score) & $28.0(4.7)$ & $28.4(1.2)$ & 0.796 \\
\hline Fugl-Meyer lower extremity (0-34) & $18.3(3.2)$ & $19.1(2.6)$ & 0.575 \\
\hline Berg balance scale (0-54) & $48.0(6.2)$ & $47.6(3.4)$ & 0.862 \\
\hline ROM of ankle dorsiflexion & $15.9(9.5)$ & $15.7(6.8)$ & 0.953 \\
\hline Ankle isometric contraction force & & $7.8(1.3)$ & 0.748 \\
\hline Dorsiflexion (N) & $14.7(6.2)$ & $14.0(4.0)$ & 0.784 \\
\hline Plantarflexion (N) & $14.3(3.0)$ & $14.8(3.4)$ & 0.752 \\
\hline Inversion (N) & $8.0(1.6)$ & $9.4(2.2)$ & 0.146 \\
\hline Eversion (N) & $7.5(2.1)$ & & 0.126 \\
\hline Walking speed (m/s) & $0.5(0.3)$ & & \\
\hline AMT, ankle muscle control training; BMI, body & mass index; K-MMES, Korean version of the mini- \\
\hline mental state examination. & & & \\
\hline Values represent mean (SD). & & & \\
\hline
\end{tabular}

Isometric contraction force of the paretic ankle

The AMT group demonstrated significant improvements in all measurements of ankle isometric strength $(P<0.01)$. There were significant between-group differences in all measurements of ankle isometric strength $(P<0.05)$.

Ankle proprioception 
Both groups displayed significant improvements in ankle proprioception 20 (AMT: $\mathrm{P}=0.046$; control: $\mathrm{P}=$ 0.031), with no between-group difference $(P=0.254$; Table 2$)$. 
Table 2

Change in outcomes between pre- and post-training

\begin{tabular}{|c|c|c|c|c|c|}
\hline \multirow[t]{2}{*}{ Measure } & & \multirow[t]{2}{*}{ Group } & \multirow[t]{2}{*}{ Pre } & \multirow[t]{2}{*}{ Post } & Between \\
\hline & & & & & $P$ value \\
\hline \multirow[t]{8}{*}{$\begin{array}{l}\text { Ankle isometric contraction } \\
\text { force }(\mathrm{N})\end{array}$} & \multirow[t]{2}{*}{ Dorsiflexion } & AMT & $\begin{array}{l}14.7 \\
(6.2)\end{array}$ & $\begin{array}{l}22.3 \\
(11.1)^{\star}\end{array}$ & \multirow[t]{2}{*}{0.034} \\
\hline & & control & $\begin{array}{l}14.0 \\
(3.9)\end{array}$ & $\begin{array}{l}14.0 \\
(6.6)\end{array}$ & \\
\hline & \multirow[t]{2}{*}{ Plantarflexion } & AMT & $\begin{array}{l}14.3 \\
(3.0)\end{array}$ & $\begin{array}{l}26.5 \\
(10.6)^{\star}\end{array}$ & \multirow[t]{2}{*}{0.003} \\
\hline & & control & $\begin{array}{l}14.8 \\
(3.3)\end{array}$ & $\begin{array}{l}15.1 \\
(6.0)\end{array}$ & \\
\hline & \multirow[t]{2}{*}{ Inversion } & AMT & $8.0(1.6)$ & $\begin{array}{l}15.6 \\
(4.7)^{*}\end{array}$ & \multirow[t]{2}{*}{$<0.001$} \\
\hline & & control & $9.3(2.1)$ & $9.8(2.9)$ & \\
\hline & \multirow[t]{2}{*}{ Eversion } & AMT & $7.5(2.1)$ & $\begin{array}{l}17.2 \\
(8.7)^{*}\end{array}$ & \multirow[t]{2}{*}{0.004} \\
\hline & & control & $7.8(1.2)$ & $8.3(1.9)$ & \\
\hline \multirow[t]{2}{*}{ Ankle proprioception $\left(^{\circ}\right)$} & & AMT & $\begin{array}{l}21.6 \\
(9.2)\end{array}$ & $\begin{array}{l}11.3 \\
(7.7)^{\star}\end{array}$ & \multirow[t]{2}{*}{0.254} \\
\hline & & control & $\begin{array}{l}14.2 \\
(4.8)\end{array}$ & $\begin{array}{l}10.0 \\
(2.8)^{*}\end{array}$ & \\
\hline \multirow{2}{*}{\multicolumn{2}{|c|}{ Fugl-Meyer lower extremity (score) }} & AMT & $\begin{array}{l}18.3 \\
(3.2)\end{array}$ & $\begin{array}{l}25.8 \\
(4.5)^{*}\end{array}$ & \multirow[t]{2}{*}{0.001} \\
\hline & & control & $\begin{array}{l}19.1 \\
(2.6)\end{array}$ & $\begin{array}{l}21.5 \\
(2.9)^{\star}\end{array}$ & \\
\hline \multirow[t]{2}{*}{ Berg balance scale (score) } & & AMT & $\begin{array}{l}48.0 \\
(6.2)\end{array}$ & $\begin{array}{l}52.4 \\
(3.9)^{*}\end{array}$ & \multirow[t]{2}{*}{0.214} \\
\hline & & control & $\begin{array}{l}47.6 \\
(3.3)\end{array}$ & $\begin{array}{l}50.2 \\
(2.6)^{\star}\end{array}$ & \\
\hline Walking speed (m/s) & & AMT & $\begin{array}{l}0.99 \\
(0.31)\end{array}$ & $\begin{array}{l}1.22 \\
(0.33)^{\star}\end{array}$ & 0.090 \\
\hline \multicolumn{6}{|l|}{ AMT, ankle muscle training. } \\
\hline Values represent mean (SD). & & & & & \\
\hline
\end{tabular}


AMT, ankle muscle training.

Values represent mean (SD).

$* \mathrm{P}<0.05$, within group comparison.

Balance and gait

Both groups exhibited significant improvements in FM-L scores $(P<0.05)$, with between-group differences $(P=0.001)$. Additionally, both groups achieved significant improvements in BBS scores $(P<0.05)$, with no between-group difference $(P=0.214)$. The AMT group displayed significant improvements in maximum walking speed $(P=0.022)$, with no between-group difference $(P=0.090)$. There was a significant betweengroup difference in stance-phase $\mathrm{Cl}$ during gait (Fig. 3; $\mathrm{P}=0.041$ ).

\section{Discussion}

This study successfully showed the effectiveness of comprehensive feedback-controlled biaxial ankle movement training on ankle motor and sensory functions, and demonstrated the increased ankle muscle strength, and the improved ankle muscle efficiency during gait, compared to control group with conventional therapy.

In stroke patients, muscle weakness has been implicated in deficient physical activity on one side of the body and this deficit is more pronounced distally than proximally [23]. A previous study showed that the residual strength following stroke was 37 percent for ankle plantar flexion, 45 percent for ankle dorsiflexion, 51 percent for knee extension, 53 percent for knee flexion, 64 percent for hip extension, and 68 percent for hip flexion [23]. The ankle muscle has been implicated as the greatest contributor to functional gait or balance performance in chronic stroke patients. Although lower extremity strengthening programs have revealed positive effects on strength and functional performance for stroke patients, targeted strengthening training for the distal part of lower extremities is required.

There are few targeted training programs or devices that strengthen or educate effectively the distal part of ankle muscles [13-15]. As a representative example, Anklebot used a target-based video game to repeat paretic ankle dorsiflexion and plantarflexion a total of 560 times per hour in a sitting position. This type of temporal symmetry [13], and step length symmetry [13], only for moderate to high functional level ankle training showed positive effects on ankle performance, including velocity [14], smoothness [14], and accuracy [14] as well as gait performance, include increased heel touch [14], individuals [15]. As there is a need to focus on more effective ankle strengthening training rather than simple repetitive exercise for stroke patients, understanding motor control will help to develop an ankle control method. To control movement, the nervous system integrates multimodal sensory information (both from the external environment and proprioception) and elicits the necessary signals to activate muscle movement. Motor 
control relies on sensory feedback to control movement using a continuous process of accessing sensory information about performance and specific sensory input from the environment to achieve more accurate motion performance [24]. Therefore, this study incorporated three training steps into the training method, reflecting the integrated sensory and motor interventions in stroke patients with various functional levels to improve ankle motor control.

According to the AMT protocol applied in this study, the passive stretching training step provided repetitive bi-axial passive ROM movement. A previous study reported that repetitive bi-axial passive ROM exercises can change the biomechanical properties of the ankle, including ankle stiffness and ankle passive ROM, resulting in improved walking performance over an uneven surface for chronic stroke patients [16]. Passive ankle stretching methods commonly influence the excitability of lower motor neurons, reduce joint stiffness and increase muscle and soft tissue extensibility, thereby providing sensory information associated with movement of the ankle muscles [25]. Muscle contraction to match movement provides information about the ankle movement direction, range, and force through visual feedback. This step allows stroke patients to repeat movement control and movement modification through visual feedback, contracting the paralyzed ankle muscles to complete the targeted movement. This multisensory feedback is important for motor learning by reestablishing the sensorimotor loop that is disrupted by stroke, because synchronization between sensory and motor information facilitates neural plasticity [26]. The final active-resistive strengthening step was important in reinforcing ankle performance through motor learning and muscle strengthening and involved individual adjustment of the difficulty level of tasks by modifying the amount of resistance and ROM. Another characteristic of ankle training in this study was the addition of diagonal movements that are practically applicable in daily activities, including walking and simple ankle biaxial movement tasks [27]. AMT tried to reproduce the actual complex ankle movements according to the anatomical characteristics of the ankle joint. Therefore, the AMT protocol presented in this study may have a positive effect on motor control and muscle strength regaining of paretic ankles in stroke patients.

Enhanced ankle strength through the AMT affects ankle motor control during gait. The ankle complex is not a simple hinged structure but functionally comprises of the ankle (talocrural) and subtalar (talocalcaneal) joint axes [28]. In particular, because of the oblique nature of the subtalar joint axis [29], plantarflexion is coupled to supination, and inversion and dorsiflexion are coupled to pronation and eversion in an orthogonal coordinate system [16]. Therefore, the increase in overall ankle muscle strength, including ankle invertor and evertor, as well as ankle dorsiflexor and ankle plantarflexor, is attributed to the fact that the ankle training device, which reflects the anatomical structure of ankle joint, effectively strengthened the muscles around the ankle by generating ankle movements in various directions. Additionally, it improved the ability of ankle muscle control, which affects gait parameters. Although the strength of the hip flexor and that of the knee extensor of the hemiplegic limb are still the most important factors determining comfortable or fast walking speed, the strength of ankle muscle is also closely related to walking speed. The power of ankle plantarflexor is a major kinematic factor that determines walking speed by producing forward propulsion, decreasing step length, and affecting gait symmetry [5, 30]. Proper ankle dorsiflexor force prevents foot drop and is properly controlled during the gait cycle as an 
antagonist of the ankle plantarflexor [31, 32]. Ankle muscles also affect walking speed by interacting with other muscles of lower extremities such as knee hyperextension related to shock absorption, decrease in step length, and compensatory movement such as hip hiking to prevent foot drop [33,34]. Stability of the distal part due to proper control of ankle muscles contributes to improved gait performance by assisting the movement of the proximal knee and hip.

Based on the relative agonist and antagonist muscle activity during walking, ankle muscle efficiency has important implications for walking efficiency. Paretic ankle muscle co-activation, which represents the activity ratio of agonist and antagonist muscles in stance phase, contributes to the compensation strategy, postural control, and regulation of joint stiffness [35, 36]. Excessive muscle co-activation can even cause severe joint stiffness, which reduces the degree of freedom [37]. Decrease in ankle cocontraction during the stance phase after AMT showed the possibility of improvements in gait parameters, including walking speed [38], an increased energetic cost [39], and a low peak plantar flexor moment on the paretic side in stroke patients [38]. Although comparative EMG analysis related to muscle activity is difficult through normalization of individual maximum muscle activity, the reduction of $\mathrm{Cl}$ in the group comparison of individuals before and after change values shows that the agonist and antagonist effect energy efficiently.

Therefore, the ankle training method provided in this study, which includes ankle passive stretching, directional perceiving, and active-resistive strength training steps, is an effective ankle rehabilitation technique that improves the quality of gait functions, with a focus on gait muscle efficiency and ankle muscle control in chronic stroke patients. This study is limited by the number of training repetitions, which varied according to the functional ability of each individual (strength and sensory functions). In additional studies, it is necessary to establish the intensity, frequency and duration of the appropriate training protocol according to the functional level of each individual.

\section{Conclusions}

Our stroke ankle rehabilitation protocol, called AMT, was able to induce initial results indicated that the training improved ankle muscle strength and walking speed in stroke patients. In addition, a significant change in muscle efficiency during gait of the patients was demonstrated compared to control group with conventional therapy. This work, therefore, emphasized the significance of comprehensive ankle training with stretching, strengthening and enhancing controllability on gait recovery in chronic stage of stroke survivors.

\section{Abbreviations}

AMT

ankle movement training

FM-L

Fugl-Meyer lower extremity 
BBS

Berg balance scale

$\mathrm{Cl}$

co-contraction index

MRC

medical research council

ROM

range of motion

10MWT

10-meter walking test

\section{Declarations}

\section{Ethics approval and consent to participate}

This randomized controlled pilot study was approved by the local Institutional Review Board at National Rehabilitation Center, and all participants enrolled in this study submitted signed informed consent form.

\section{Consent for publication}

$\mathrm{N} / \mathrm{A}$

\section{Availability of data and materials}

The datasets used and analyzed in this study are available from the corresponding author on reasonable request.

\section{Competing interests}

The Authors declare that there is no conflict of interest.

\section{Funding}

This work was supported by Translational Research Project for Rehabilitation Robots, Korea National Rehabilitation Center, Ministry of Health \& Welfare, South Korea (\#NRCTR-IN18003).

\section{Authors' contributions}

All authors made a substantial contribution of the concept of this study, data collection, data analysis, interpretation, and drafting the manuscript. Authors provided approval of final version of manuscript.

\section{Acknowledgements}

The authors would like to appreciate all subjects for their supports and participation. A special thanks to Dr. Dohoon Koo for his assistance on motion capturing during the experiments. 


\section{References}

[1] Karakaya MGr, Rutbil H, Akpinar E, Yildirim A, Karakaya iCi. Effect of ankle proprioceptive training on static body balance. Journal of physical therapy science 2015;27:3299-302.

[2] An C-M, Won J-I. Effects of ankle joint mobilization with movement and weight-bearing exercise on knee strength, ankle range of motion, and gait velocity in patients with stroke: a pilot study. Journal of physical therapy science 2016;28:689-94.

[3] Teasell RW, McRae MP, Foley N, Bhardwaj A. Physical and functional correlations of ankle-foot orthosis use in the rehabilitation of stroke patients. Archives of physical medicine and rehabilitation 2001;82:1047-9.

[4] Meinders M, Gitter A, Czerniecki J. The role of ankle plantar flexor muscle work during walking. Scandinavian journal of rehabilitation medicine 1998;30:39-46.

[5] Bowden MG, Balasubramanian CK, Neptune RR, Kautz SA. Anterior-posterior ground reaction forces as a measure of paretic leg contribution in hemiparetic walking. Stroke 2006;37:872-6.

[6] Lamontagne A, Malouin F, Richards CL. Locomotor-specific measure of spasticity of plantarflexor muscles after stroke. Archives of Physical Medicine and Rehabilitation 2001;82:1696-704.

[7] Schmidt RA, Lee TD, Winstein C, Wulf G, Zelaznik HN. Motor control and learning: A behavioral emphasis. Human kinetics; 2018.

[8] Hillier S, Immink M, Thewlis D. Assessing proprioception: a systematic review of possibilities. Neurorehabilitation and neural repair 2015;29:933-49.

[9] Mayr A, Kofler M, Quirbach E, Matzak H, Fröhlich K, Saltuari L. Prospective, blinded, randomized crossover study of gait rehabilitation in stroke patients using the Lokomat gait orthosis. Neurorehabilitation and neural repair 2007;21:307-14.

[10] Schmidt H, Werner C, Bernhardt R, Hesse S, Krüger J. Gait rehabilitation machines based on programmable footplates. Journal of neuroengineering and rehabilitation 2007;4:2.

[11] Nadeau SE, Wu SS, Dobkin BH, Azen SP, Rose DK, Tilson JK, Cen SY, Duncan PW, Team LI. Effects of task-specific and impairment-based training compared with usual care on functional walking ability after inpatient stroke rehabilitation: LEAPS Trial. Neurorehabilitation and neural repair 2013;27:370-80.

[12] Forrester LW, Roy A, Krebs HI, Macko RF. Ankle training with a robotic device improves hemiparetic gait after a stroke. Neurorehabilitation and neural repair 2011;25:369-77.

[13] Forrester LW, Roy A, Krywonis A, Kehs G, Krebs HI, Macko RF. Modular ankle robotics training in early subacute stroke: a randomized controlled pilot study. Neurorehabilitation and neural repair 2014;28:678- 
87.

[14] Roy A, Forrester LW, Macko RF. Short-term ankle motor performance with ankle robotics training in chronic hemiparetic stroke. Journal of Rehabilitation Research \& Development 2011;48:417-30.

[15] Chang JL, Lin RY, Saul M, Koch PJ, Krebs HI, Volpe BT. Intensive seated robotic training of the ankle in patients with chronic stroke differentially improves gait. NeuroRehabilitation 2017;41:61-8.

[16] Kim H, Cho S, Lee H. Effects of Passive Bi-axial Ankle Stretching while Walking on Uneven Terrains in Older Adults with Chronic Stroke. Journal of Biomechanics 2019.

[17] Holden MK, Gill KM, Magliozzi MR, Nathan J, Piehl-Baker L. Clinical gait assessment in the neurologically impaired: reliability and meaningfulness. Physical therapy 1984;64:35-40.

[18] Contu S, Hussain A, Kager S, Budhota A, Deshmukh VA, Kuah CW, Yam LH, Xiang L, Chua KS, Masia L. Proprioceptive assessment in clinical settings: Evaluation of joint position sense in upper limb poststroke using a robotic manipulator. PloS one 2017;12:e0183257.

[19] Duncan PW, Propst M, Nelson SG. Reliability of the Fugl-Meyer assessment of sensorimotor recovery following cerebrovascular accident. Physical therapy 1983;63:1606-10.

[20] Downs S, Marquez J, Chiarelli P. The Berg Balance Scale has high intra-and inter-rater reliability but absolute reliability varies across the scale: a systematic review. Journal of physiotherapy 2013;59:93-9.

[21] Kellis E, Arabatzi F, Papadopoulos C. Muscle co-activation around the knee in drop jumping using the co-contraction index. Journal of Electromyography and Kinesiology 2003;13:229-38.

[22] Forrester LW, Roy A, Hafer-Macko C, Krebs HI, Macko RF. Task-specific ankle robotics gait training after stroke: a randomized pilot study. Journal of neuroengineering and rehabilitation 2016;13:51.

[23] Adams R, Gandevia S, Skuse N. The distribution of muscle weakness in upper motoneuron lesions affecting the lower limb. Brain 1990;113:1459-76.

[24] Adams JA. A closed-loop theory of motor learning. Journal of motor behavior 1971;3:111-50.

[25] James SF, M. Contractures in orthopaedic and neurological conditions: a review of causes and treatment. Disability and rehabilitation 2001;23:549-58.

[26] Johansson B. Current trends in stroke rehabilitation. A review with focus on brain plasticity. Acta Neurologica Scandinavica 2011;123:147-59.

[27] Abreu R, Lopes AA, Sousa AS, Pereira S, Castro MP. Force irradiation effects during upper limb diagonal exercises on contralateral muscle activation. Journal of Electromyography and Kinesiology 2015;25:292-7. 
[28] Wu G, Siegler S, Allard P, Kirtley C, Leardini A, Rosenbaum D, Whittle M, D D'Lima D, Cristofolini L, Witte $\mathrm{H}$. ISB recommendation on definitions of joint coordinate system of various joints for the reporting of human joint motion-part I: ankle, hip, and spine. Journal of biomechanics 2002;35:543-8.

[29] Jastifer JR, Gustafson PA. The subtalar joint: biomechanics and functional representations in the literature. The foot 2014;24:203-9.

[30] Awad LN, Reisman DS, Kesar TM, Binder-Macleod SA. Targeting paretic propulsion to improve poststroke walking function: a preliminary study. Archives of physical medicine and rehabilitation 2014;95:840-8.

[31] Dorsch S, Ada L, Canning CG, Al-Zharani M, Dean C. The strength of the ankle dorsiflexors has a significant contribution to walking speed in people who can walk independently after stroke: an observational study. Archives of physical medicine and rehabilitation 2012;93:1072-6.

[32] Ng SS, Hui-Chan CW. Contribution of ankle dorsiflexor strength to walking endurance in people with spastic hemiplegia after stroke. Archives of physical medicine and rehabilitation 2012;93:1046-51.

[33] Morris M, Matyas T, Bach T, Goldie P, editor^editors. The effect of electrogoniometric feedback on knee hyperextension following stroke. Proceedings of the 11 th International Congress of the World Confederation for Physical Therapy. London; 1991. p 469-71.

[34] Cooper A, Alghamdi GA, Alghamdi MA, Altowaijri A, Richardson S. The relationship of lower limb muscle strength and knee joint hyperextension during the stance phase of gait in hemiparetic stroke patients. Physiotherapy research international 2012;17:150-6.

[35] Falconer K, Winter D. Quantitative assessment of co-contraction at the ankle joint in walking. Electromyography and clinical neurophysiology 1985;25:135-49.

[36] Rosa MCN, Marques A, Demain S, Metcalf CD. Lower limb co-contraction during walking in subjects with stroke: A systematic review. Journal of Electromyography and Kinesiology 2014;24:1-10.

[37] Tucker MG, Kavanagh JJ, Barrett RS, Morrison S. Age-related differences in postural reaction time and coordination during voluntary sway movements. Human movement science 2008;27:728-37.

[38] Lamontagne A, Richards CL, Malouin F. Coactivation during gait as an adaptive behavior after stroke. Journal of Electromyography and Kinesiology 2000;10:407-15.

[39] Detrembleur C, Dierick F, Stoquart G, Chantraine F, Lejeune T. Energy cost, mechanical work, and efficiency of hemiparetic walking. Gait \& posture 2003;18:47-55.

\section{Figures}


Assessed for eligibility $(n=27)$

\section{Excluded $(n=2)$}

- Unable to walk $(n=1)$

- Unable to follow instructions $(n=1)$

Randomized ( $n=25)$

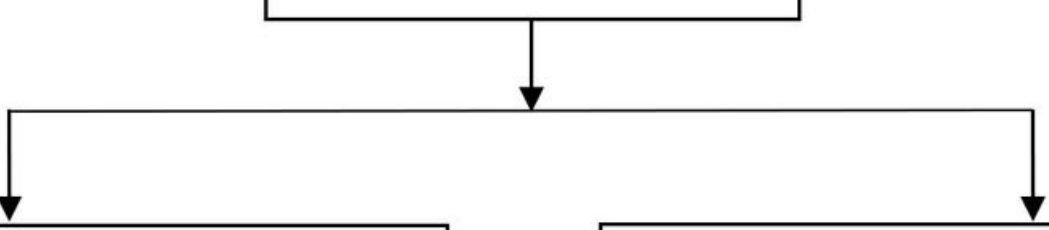

Allocated to intervention $(n=13)$

- Received the allocated intervention $(n=13)$

- Did not receive the allocated intervention $(n=0)$

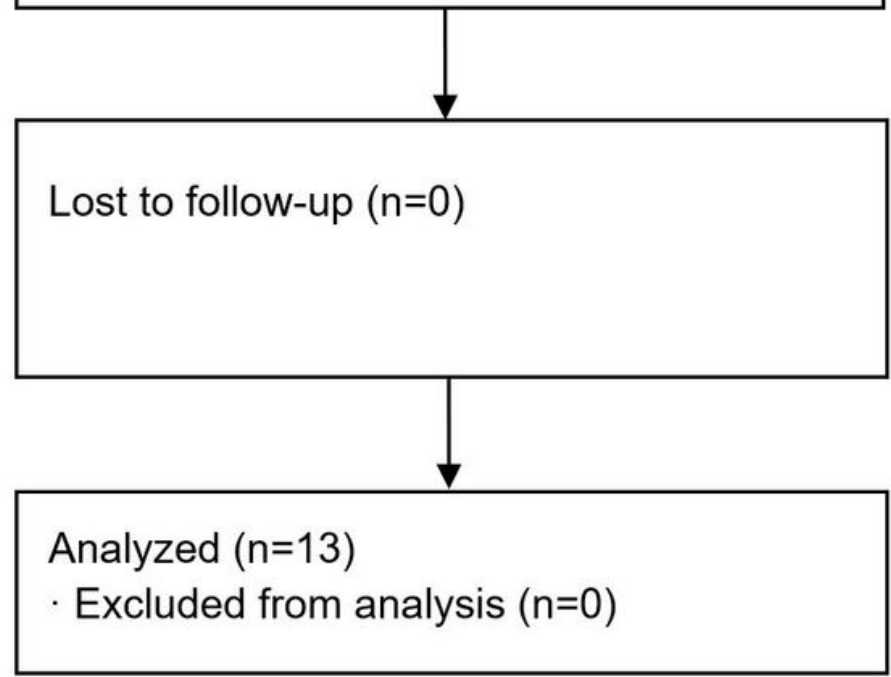

Allocated to intervention (control) $(n=12)$

- Received the allocated intervention $(\mathrm{n}=12)$

- Did not receive the allocated intervention $(n=0)$

Lost to follow-up ( $\mathrm{n}=2$ )

- Unable to return after hospital discharge $(n=2)$

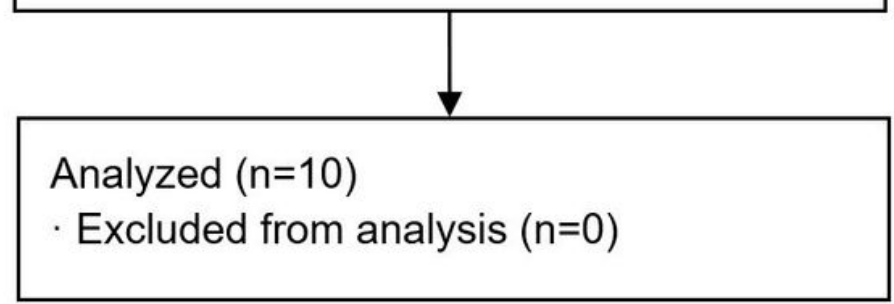

\section{Figure 1}

A single blinded, parallel-group, randomized controlled trial with blinding of assessors and concealed allocation was conducted. A person not involved in the trial created a blocked random allocation scheduled for 20 participants using Microsoft Excel. Blocking ensured equal numbers of participants in the experimental and control groups. Participants in the experimental group underwent ankle movement training (AMT) consisting of passive stretching, contraction to match movement, and active-resistive strengthening using visual feedback for four weeks. Participants in control group underwent usual ankle relative physical therapy for four weeks. All participants were re-assessed at the end of the four-week intervention period. 


\section{A. Sequence of training protocol}

\section{Step 1: Passive Stretching}

Bi-axial ankle stretching \& passive ROM (range of motion) exercise

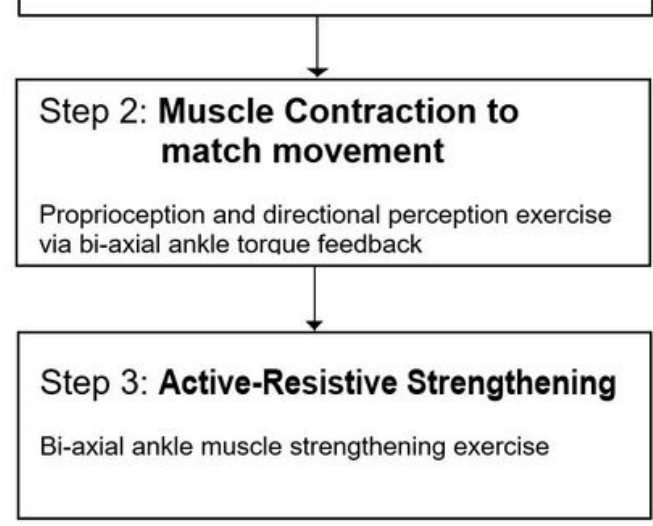

B. Ankle muscle control training using visual feedback

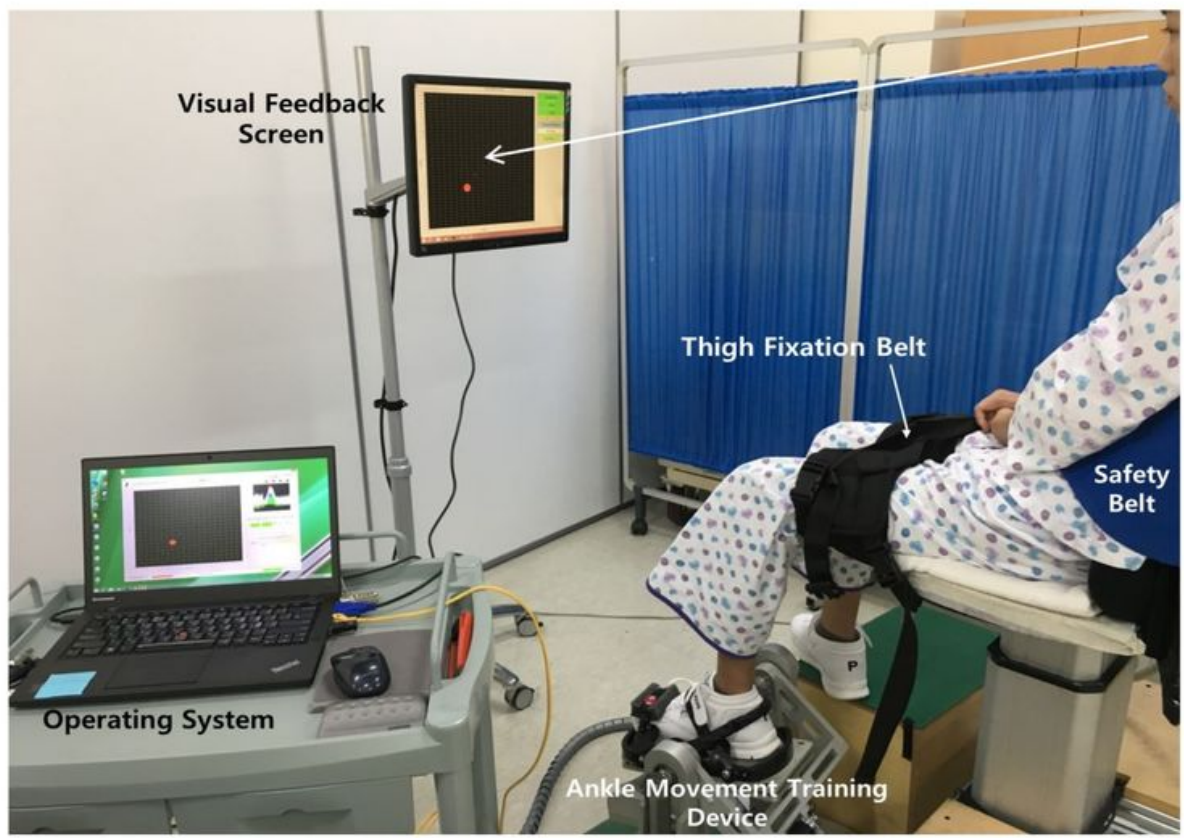

Figure 2

A monitor providing visual feedback was placed at eye level of the participant (2-B). The AMT protocol consisted of three steps (2-A). 


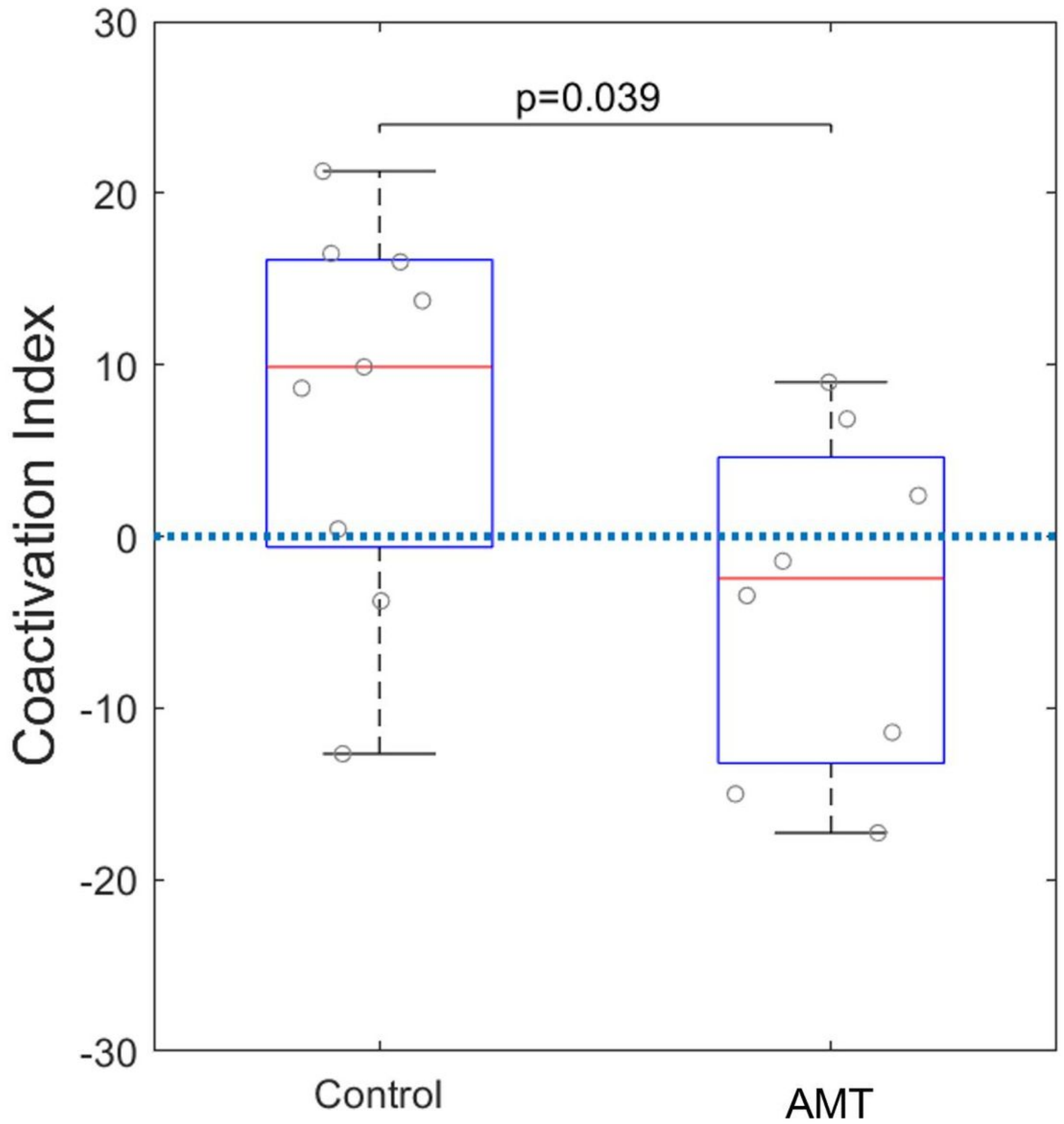

Figure 3

Both groups exhibited significant improvements in FM-L scores $(P<0.05)$, with between-group differences $(P=0.001)$. Additionally, both groups achieved significant improvements in BBS scores $(P<0.05)$, with no between-group difference $(P=0.214)$. The AMT group displayed significant improvements in maximum walking speed $(P=0.022)$, with no between-group difference $(P=0.090)$. There was a significant betweengroup difference in stance-phase $\mathrm{Cl}$ during gait $(\mathrm{P}=0.041)$. 


\section{Supplementary Files}

This is a list of supplementary files associated with this preprint. Click to download.

- CONSORTChecklistv2.doc

- CONSORTFlowDiagram.docx

- AMTVIDEOclips.wmv 